\title{
VARIAÇÃO MORFOLÓGICA EM DENTES ROSTRAIS DO ESPADARTE EXTINTO Atlanticopristis equatorialis PEREIRA \& MEDEIROS, 2008
}

\author{
Lays S. de Oliveira Silva ${ }^{1}$, Jorge L. S. Nunes ${ }^{2} \&$ Manuel Alfredo Medeiros $^{3}$ \\ ${ }^{1}$ Centro de Pesquisa de História Natural e Arqueologia do Maranhão, Rua do Giz 59, Praia Grande, CEP 65010-680, \\ São Luís, MA, Brasil. \\ ${ }^{2}$ Universidade Federal do Maranhão, Departamento de Oceanografia e Limnologia. Avenida dos Portugueses 1966, \\ Cidade Universitária do Bacanga, CEP 65080-805, São Luís, MA, Brasil. \\ ${ }^{3}$ Universidade Federal do Maranhão, Departamento de Biologia. Avenida dos Portugueses 1966, Cidade Universitária \\ do Bacanga, CEP 65080-805, São Luís, MA, Brasil.
}

\begin{abstract}
RESUMO
As raias-espadarte Esclerorhynchidae (Batoidea) fazem parte de um grupo extinto monofilético que é bem registrado no Cretáceo. Para avaliar a variação morfológica intraespecífica de uma espécie do Cretáceo, os dentes rostrais referidos a Atlanticopristis equatorialis Pereira \& Medeiros, 2008 foram analisados e seus caracteres morfológicos medidos e comparados. A coroa dos dentes rostrais exibe margens anterior e posterior multidenticuladas ( 2 a 5 farpas em cada margem). Em algumas amostras, pequenas protuberâncias próximas à base podem ser consideradas dentículos (farpas) vestigiais. Nervuras enameloides (costelas) extensas são vistas nas superfícies dorsal e ventral da coroa; as costelas centralizadas são paralelas e confinadas à porção mais proximal da coroa, com as periféricas formando ângulos progressivamente maiores em relação ao eixo longitudinal do dente, resultando em uma aparência de leque. Foi observada uma variação de 6 a 13 costelas. A variação observada no pedúnculo é menos útil em relação à taxonomia, uma vez que serve como uma "raiz" para os dentes e muitos padrões diferentes de superfícies rugosas podem ser fixados por ligamentos. As características da coroa são mais úteis para distinção taxonômica e suas medidas indicam variação intraespecífica em $A$. equatorialis.

Palavras-chave: Batoidea, Sclerorhynchidae, Formação Alcântara, Cretáceo.
\end{abstract}

\begin{abstract}
Sclerorhynchid sawfishes (Batoidea) are part of a monophyletic extinct group that is well recorded in the Cretaceous. In order to evaluate the intraspecific morphological variation of a Cretaceous species, rostral teeth referred to Atlanticopristis equatorialis Pereira \& Medeiros, 2008 were analyzed and their morphological characters measured and compared. The crown of the rostral teeth exhibits multibarbed anterior and posterior margins ( 2 to 5 barbs on each margin). In some specimens tiny protuberances close to the base might be considered as vestigial barbs. Extensive enameloid ribbing are seen on the dorsal and ventral surfaces of the crown; centralized ribs are parallel and confined to the more proximal portion of the crown, with peripheral ribs forming progressively wider angles in relation to the longitudinal axis of the tooth, resulting in a fan-like appearance. A variation from 6 to 13 ribs have been observed. The variation seen in the peduncle is the less useful regarding taxonomic importance since it serves as a "root" to the teeth and many different patterns of rugose surfaces are able to be attached by ligaments. The crown features are more useful for taxonomic distinction and its measures indicate intraspecific variation in A. equatorialis.

Key words: Batoidea, Sclerorhynchidae, Alcântara Formation, Cretaceous.
\end{abstract}

\section{INTRODUÇÃO}

Os Sclerorhynchidae formam um diversificado grupo monofilético de elasmobrânquios extintos que viveram durante o Cretáceo, entre o Barremiano (129 m.a) e Maastrichtiano (65 m.a) (Kriwet \& Kussius 2001; Underwood 2006; Almafitano et al. 2017). Estes elasmobrânquios Batomorphii, em sua maioria, alcançavam cerca de $100 \mathrm{~cm}$ de comprimento total e viviam em ambientes de água doce ou em ambientes marinhos tropicais de águas rasas (Capetta1980; 1987; Branch \& Mosley 1997), embora algumas espécies frequentassem ambientes de mar aberto (Smith et al. 2015). Possuíam um rostrum cartilaginoso hipertrofiado dorsoventralmente achatado, contendo inúmeras ampolas de Lorenzini na porção ventral e margeado por numerosos dentes rostrais (Wueringer et al. 2011). 
O rostrum dos Sclerorhynchidae é semelhante às mesmas estruturas presentes nas espécies ainda viventes de raias espadarte (Pristidae) e de tubarões espadartes (Pristiophoridae) (Cappetta 1974, 1980; Wueringer et al. 2009). A presença do rostrum nestes grupos é caracterizada como uma adaptação convergente para alimentação e defesa (Kriwet 2004). Nos Pristidae atuais é usado para vasculhar o fundo lamoso e desalojar possíveis presas, assim como o utilizam para golpear peixes, aprisionandoos nos dentículos (Feitosa et al. 2017). O peixe é posteriormente solto através de fricção do rostrum no fundo do mar, o que possibilita a ingestão da presa (Kirkland \& Martínez 2002).

A similaridade morfológica entre espadartes atuais e extintos propicia inferências em função da sua morfologia funcional, pois acredita-se que as raias espadarte extintas exploravam o mesmo nicho ecológico que as atuais, utilizando o rostrum para alimentação e defesa de forma idêntica (Sternes \& Shimada 2018). Outras informações também indicam o mesmo modo de vida, tais como a similaridade dos ambientes explorados (Feitosa et al. 2017) e as disposições do sistema de ampolas de Lorenzini utilizado particularmente em águas turvas (Wueringer et al. 2011; Feitosa et al. 2019; Wosnick et al. 2019).

Por outro lado, as divergências em detalhes morfológicos do rostrum parecem indicar o surgimento desta adaptação, de forma independente, pelo menos três vezes em diferentes linhagens evolutivas dos elasmobrânquios (Wueringer et al. 2009). Enquanto os Sclerorhynchidae possuíam dentes fixados no rostrum por meio de ligamentos de tecido conjuntivo, que permitia substituição constante dos mesmos, os Pristidae possuem dentes inseridos em alvéolos sem a substituição ao longo da vida. Os tubarões espadartes atuais (Pristiophoridae) também têm seus dentes rostrais fixados por ligamentos. Esta é considerada uma condição primitiva semelhante aos Sclerorhynchidae extintos (ver Slaughter \& Springer 1968; Cappetta 1974, 1980, 1987; Kirkland \& Martínez 2002; Gomes et al. 2012).

Estudos paleontológicos sobre os Chondrichthyes são limitados devido à dificuldade de fossilização de estruturas cartilaginosas. Portanto, os registros de exemplares completos são excepcionalmente raros (Arambourg 1940). Alguns dos melhores registros fósseis de Sclerorhynchidae foram encontrados em rochas depositadas em ambientes marinhos no Líbano e primeiramente estudados por Smith Woodward, no final do século XIX (Cappetta 1980; 1987). Os dentes bucais, dentículo dérmicos, dentes rostrais e rostrum são as estruturas mineralizadas mais encontradas no registro fóssil, possuindo uma destacada importância taxonômica para as formas extintas (Sternes \& Shimada 2018). Desta forma, os Sclerorhynchidae são identificados por meio de características diagnósticas da coroa e pedúnculo dos dentes rostrais. A coroa apresenta variações entre os 24 gêneros descritos. Normalmente é deprimida, coberta por substância semelhante a esmalte (enamelóide), com porção distal geralmente afiada e frequentemente possuindo dentículos (farpas). O pedúnculo apresenta extremidade proximal côncava e sulcos irregulares nas laterais para a fixação dos ligamentos nos dentes rostrais, mantendo-os firmes na borda do rostrum (Arambourg 1940).

Três gêneros de Sclerorhynchidae já foram registrados no estado do Maranhão. Todos encontrados no Grupo Itapecuru, conjunto geológico cretáceo que aflora ao longo do vale do rio Itapecuru, na Ilha do Maranhão e em Alcântara (Rossetti \& Truckenbrodt 1997; Pedrão et al. 1993; Vicalvi \& Carvalho 2002). Dentes bucais do gênero Ischyrhiza foram encontrados na região de Coroatá (Fontes et al. 2012). Os gêneros Onchopristis e Atlanticopristis foram encontrados na Formação Alcântara (Pereira \& Medeiros 2008; Medeiros et al. 2014), depositada em ambiente estuarino (Klein \& Ferreira 1979; Pedrão et al. 1993; Dutra \& Malabarba 2001).

Atlanticopristis equatorialis foi definida com base em um pequeno conjunto de dentes rostrais. Depois de definidos os epítetos genérico e específico, em 2008, mais material foi coletado. Este conjunto de espécimes mais recentemente coletados mostra uma variação morfológica dos caracteres considerados diagnósticos por Pereira \& Medeiros (2008). É importante investigar se esta variação pode ser interpretada como dentro da amplitude de uma única espécie ou se poderia indicar mais de uma espécie dentro do gênero Atlanticopristis. Assim, o presente trabalho tem como objetivo analisar e interpretar a variação morfológica de dentes rostrais de $A$. equatorialis Pereira \& Medeiros, 2008 provenientes da Formação Alcântara (Cretáceo, Cenomaniano), Ilha do Cajual, município de Alcântara, no intuito de definir em que nível se enquadraria como elemento diagnóstico para a taxonomia.

\section{MATERIAL E MÉTODOS}

Vinte e dois dentes rostrais analisados, inteiros ou incompletos, foram coletados no afloramento Falésia do Sismito, Ilha do Cajual ( $2^{\circ} 28^{\prime} 56,31$ 's; 
$44^{\circ} 28$ '09,57'O), estado do Maranhão, Litoral Amazônico Brasileiro (Figura 1). Os exemplares foram coletados pelo método de peneiramento de sedimentos e métodos tradicionais de retirada de fósseis diretamente da rocha matriz com ferramentas manuais. Os exemplares foram tombados e armazenados na coleção de vertebrados do Centro de Pesquisa em História Natural e Arqueologia do Maranhão (CPHNAMA). Posteriormente, realizouse a documentação fotográfica sob ampliação em lupa Zeiss Axio Scope e medição dos caracteres por meio de um paquímetro Messen 150 x $6 \mathrm{~mm}$, a fim de descrever a variação presente na amostra. Devido ao fato de grande parte dos dentes rostrais estarem incompletos, a medição de caracteres como o comprimento total do dente, número de farpas na borda anterior e posterior e número de estrias na face 1 e 2 não puderam ser mensurados em sua totalidade em alguns exemplares.

Com base no trabalho de Pereira \& Medeiros (2008) 13 caracteres foram mensurados: comprimento total do dente $(\mathrm{CT})$, comprimento da coroa (CC), número de farpas na borda anterior (FA), número de farpas na borda posterior (FP), número de estriais na face 1 (EF1), número de estriais na face 2 (EF2), largura ântero-posterior basal do pedúnculo (LB), espessura dorso-ventral basal do pedúnculo (EB), largura anteroposterior do pedúnculo - limite superior (LS), espessura dorso-ventral do pedúnculo - limite superior (ES) e comprimento do pedúnculo (CP). Também foram considerados os índices de constrição (IC), resultado da diferença entre LB e LS; índice de compressão do pedúnculo (ICP) e o resultado da diferença entre EB e ES. A análise de dados iniciou com a organização da matriz com os valores de medidas morfométricas de exemplares que exibiam grande parte das medidas avaliadas (16 exemplares). Porém, foi considerado apenas sete dos 13 caracteres mensurados (LB, EB, LS, ES, CP, IC e ICD). Inicialmente, os dados morfométricos foram submetidos ao fator de correção em função do tamanho, aplicando o método Burnaby para evitar os efeitos alométricos dos dentes rostrais (Klingenberg, 2016). Em seguida, a Análise de Componentes Principais (ACP) foi aplicada ao conjunto de dados com o intuito de determinar os caracteres morfológicos que descrevem as principais variações na amostra por meio do Software PAST (Paleontological Statistics, versão 3.18) (Hammer et al., 2001).

\section{RESULTADOS}

Todos os dentes rostrais apresentam coroa e pedúnculos esmaltados e deprimidos, típicos da família Sclerorhynchidae. Dentículos estão presentes na margem anterior e posterior e estrias em ambas as faces, estendendo-se do limite superior do pedúnculo até a coroa nos exemplares mais bem preservados. Tais caracteres são considerados diagnósticos para Atlanticopristis equatorialis Pereira \& Medeiros 2008.

O maior dente rostral completo da espécie possui $21 \mathrm{~mm}$ de comprimento, 4 farpas na margem anterior, 4 na margem posterior, com 8 e 9 estrias nas faces dorsal e ventral. Exibe contorno basal retangular, LB 6,4 mm e EB com 3,6 mm (exemplar 24). O menor exemplar completo, VT 1088-8 (Figura 2) apresenta $7,3 \mathrm{~mm}$ de comprimento, 2 farpas na margem anterior, 4 na margem posterior com 6

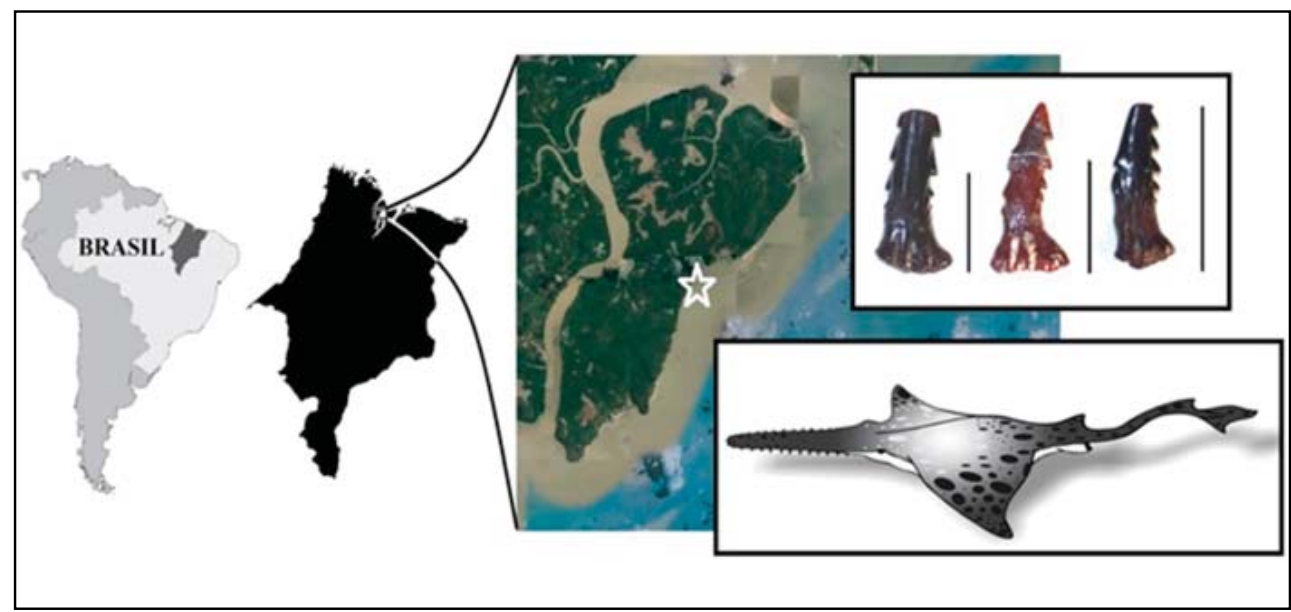

Figura 1. a) Ilha do Cajual, Maranhão, Litoral Amazônico Brasileiro e posição da Falésia do Sismito (estrela). Exemplos dos dentes rostrais estudados; da esquerda para direita: VT 1172-19, VT 1331, VT 1088-8. Escalas $=1 \mathrm{~cm}$. Desenho: Tainá Constância de França. 
estrias em uma das faces e 7 na outra, contorno basal elipsoidal, LB 2,6 mm e EB com 1,4 mm. Portanto, a quantidade máxima de farpas inequívocas, facilmente distinguíveis, é de quatro na margem anterior e cinco na margem posterior. Em alguns espécimes, pequenas protuberâncias próximas da base são consideradas como farpas vestigiais.

O padrão de ocorrência das estrias é correspondente com a descrição realizada por Pereira \& Medeiros (2008), pois, de acordo com os autores, as estriais originam-se na base da coroa, formando ângulos progressivamente maiores em relação ao eixo longitudinal do dente, exceto as estrias mais centrais que são mantidas quase paralelas, criando um padrão em leque. O comprimento das estrias centrais e laterais pode variar, como visto em VT 1172-19 e VT 1088-1 (Figura 3a, b, respectivamente).

Observou-se diferença evidente quanto à constrição no limite superior do pedúnculo (IC), possuindo variação entre 0.7 (VT 1088-8) (Figura 3c) e $3.8 \mathrm{~mm}$ (exemplar 14) (Figura 3d). No que se refere ao contorno basal do pedúnculo, também foi observada variação. Em alguns espécimes, o contorno apresenta forma retangular (exemplar 23) (Figura 3e) e em outros, elipsoidal (VT 1088-5) (Figura 3f), ou sub-retangular (exemplar 26) (Figura

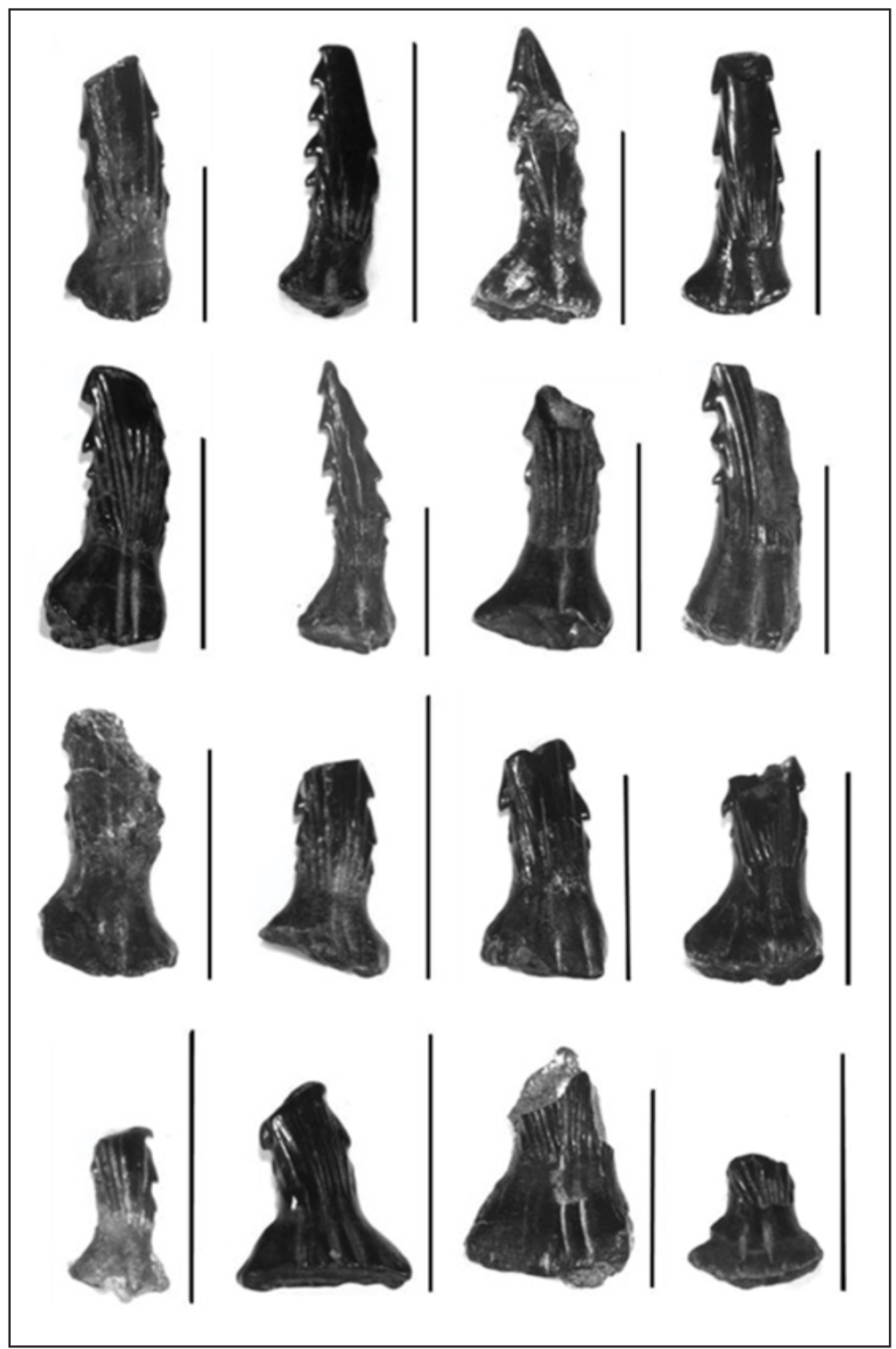

Figura 2. Da esquerda para direita: 1088-5, 1088-8, 1331, 1172-19, 1172-21, exemplares 24 e 25, 1088-1, 1088-4, 1088-9, exemplar 22, 1172-18, exemplares $12,14,23$ e 26. Escala $=1 \mathrm{~cm}$. 


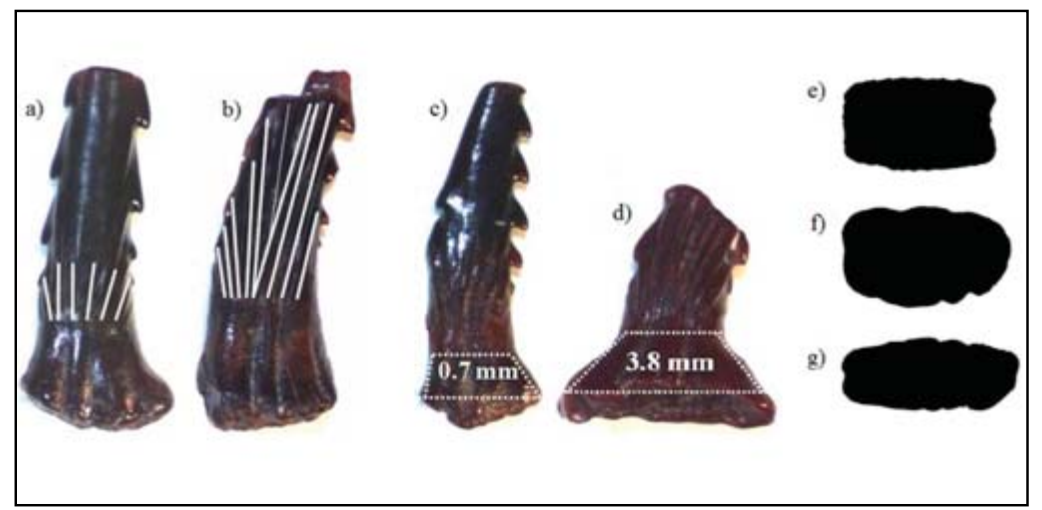

Figura 3. Variação morfológica em dentes rostrais de A. equatorialis. a) 1172-19 e b) 1088-1, variação relacionada à quantidade e ao comprimento das estrias; c 1088-8 e d) exemplar 14, variação relacionada à constrição no limite superior do pedúnculo; e) exemplar 23, f) 1088-5 e g) exemplar 26, variação relacionada à forma do pedúnculo em vista basal.

$3 \mathrm{~g}$ ); os dois últimos tipos também reportados por Pereira \& Medeiros (2008).

AAnálise de Componentes Principais mostrou que os dois primeiros eixos acumularam $92,41 \%$ da variância explicada (Eixo $1=57,09 \%$; Eixo 2 $=35,32 \%$ ) e formou quatro agrupamentos, um por quadrante (Tabela 1; Figura 4).

O Índice de Compressão Dorso-Ventral do Pedúnculo (ICD) foi a maior medida do primeiro eixo, sendo a única que apresentou valor negativo. Por outro lado, no segundo eixo as medidas Índice de Constrição da Base do Pedúnculo (IC) e Largura Ântero-Posterior da Base do Pedúnculo (LB) foram as únicas medidas positivas, sendo a primeira com maior valor no eixo (Tabela 1).

As variáveis morfológicas IC e LB descreveram o grupo formado pelo Exemplar 14 e Exemplar 25; as medidas CP, EB, ES e LS agruparam os dentes 1088-4, 1088-5 e 1172-18; a medida ICD agrupou o Exemplar 22, Exemplar 23, 1088-1, 1088-8 e 117219; por fim, as cargas negativas de CP, EB, ES e LS agruparam os demais dentes rostrais (Figura 4).

\section{DISCUSSÃO}

A sutil variação de caracteres da coroa pode estar relacionada com a posição dos dentes ao longo do rostrum ou à alometria ontogenética (ver Schaeffer, 1963; Slaughter \& Springer, 1968; Sternes \& Shimada, 2018). Em Sclerorhynchidae há registros tanto de variação de tamanho dentro de uma espécie, quanto de certa uniformidade em um único espécime (ver Welten et al., 2015).

O Índice de Constrição da Base do Pedúnculo (IC) e o Índice de Compressão Dorso-Ventral do
Pedúnculo (ICD) foram os caracteres que apresentaram as maiores cargas de variação total na Análise de Componentes Principais. Essa variação pode estar relacionada aos diversos padrões de superfície do pedúnculo que podem ser fixados diretamente no rostrum, uma vez que nos sclerorhynchídeos, os dentes eram inseridos por ligamentos. Os caracteres da coroa variaram mais discretamente e dentro do que pode seguramente ser interpretado como intraespecífico, visto que o nível de variação destes caracteres está dentro de limites esperados. Tratase claramente de mudanças que se acentuam ou se atenuam gradativamente, sem intervalos bruscos

Tabela 1- Cargas dos atributos morfológicos sobre os dois primeiros componentes principais, com indicação das variáveis mais importantes $(*)$ e que explicam a distribuição dos dentes rostrais (Veja texto para definição das siglas).

\begin{tabular}{c|c|c}
\hline & CP 1 & CP 2 \\
\hline LB & 0,1993 & 0,1767 \\
\hline EB & 0,0972 & $-0,1249$ \\
\hline LS & 0,3315 & $-0,3051$ \\
\hline ES & 0,442 & $-0,131$ \\
\hline CP & 0,1869 & $-0,1879$ \\
\hline IC & 0,01303 & $0,8752 *$ \\
\hline ICD & $-0,7813 *$ & $-0,2044$ \\
\hline Autovalores & 0,0345 & 0,0151 \\
\hline Var. explicada & $57,09 \%$ & $35,32 \%$ \\
\hline
\end{tabular}




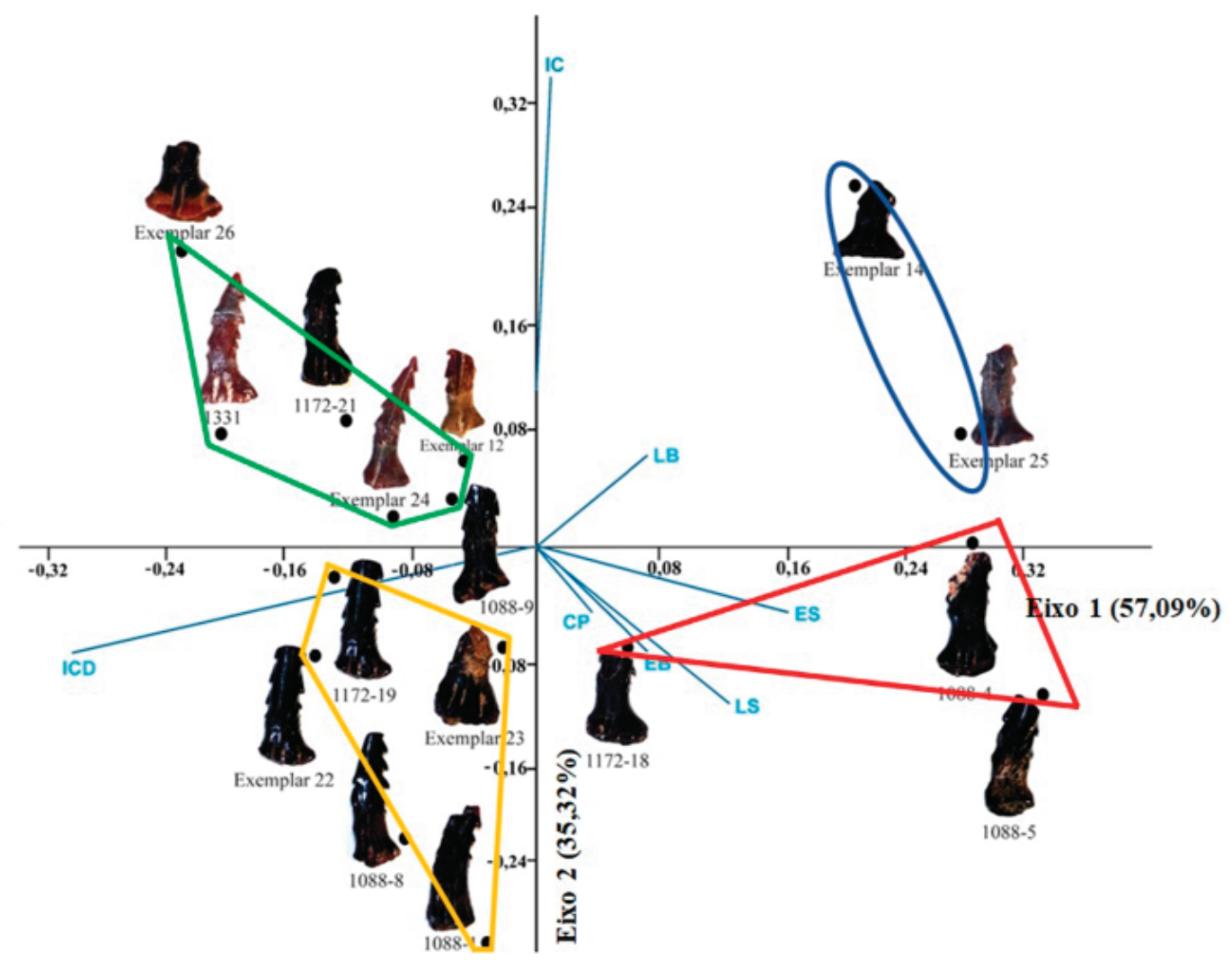

Figura 4. Análise de Componentes Principais aplicada aos dentes rostrais e aos caracteres morfológicos com seus respectivos escores. Largura ântero-posterior basal do pedúnculo (LB), espessura dorso-ventral basal do pedúnculo (EB), largura ântero-posterior do pedúnculo- limite superior (LS), espessura dorso-ventral do pedúnculo - limite superior (ES), comprimento do pedúnculo (CP). Também foram considerados os índices de constrição (IC) e Índice de compressão do pedúnculo (ICP).

entre elas, o que normalmente acontece em variação intraespecífica, intrapopulacional, ontogenética, ou mesmo variação de posição ao longo do rostrum (ver Schaeffer, 1963; Slaughter \& Springer, 1968; Sternes \& Shimada, 2018).

Com base na variação observada de caracteres e com as informações da literatura, todos os espécimes aqui amostrados e analisados podem ser seguramente atribuídos à espécie Atlanticopristis equatorialis Pereira \& Medeiros, 2008. Este estudo pode ser útil em futuras comparações de dentes rostrais pertencentes ao gênero Atlanticopristis. Exemplares que venham a ser coletados no nordeste do Brasil e que apresentem variação dentro do espectro aqui documentado devem ser considerados como pertencentes a A. equatorialis.

Considerando-se a taxonomia baseada em dentes rostrais, o gênero Onchopristis é o táxon morfologicamente mais proximamente relacionado a Atlanticopristis. Entretanto, os padrões morfológicos entre os dois gêneros são facilmente distinguíveis (ver McNulty \& Slaughter, 1962; Keyes, 1977; Capetta, 1987; Pereira \& Medeiros, 2008). Os dentes rostrais de $A$. equatorialis apresentam uma morfologia ímpar, com farpas nas duas margens e ornamentação exclusiva.

A associação dos dentes rostrais de $A$. equatorialis a fósseis de formas continentais como peixes dulcícolas (e.g. Mawsonia, Lepidotes, peixes pulmonados ceratodontídeos), crocodilos, dinossauros e pterossauros, indica claramente que se tratava de um elasmobrânquio capaz de frequentar águas doces, dentro do domínio estuarino registrado pela Formação Alcântara (Medeiros et al., 2014; Klein \& Ferreira, 1979; Pedrão et al., 1993), mas possivelmente frequentando águas costeiras em parte do seu ciclo de vida (Pereira \& Medeiros, 2008). Entretanto, não é possível saber com certeza se seu ciclo de vida consistia em diferentes padrões de uso de habitat, como acontece com os Pristis atuais (Feitosa et al., 2017), ou em função de plasticidade fisiológica, como atualmente observado em tubarões 
da espécie Carcharhinus leucas (Feitosa et al., 2016). As duas condições são perfeitamente aceitáveis, pois estes padrões são registrados para as espécies viventes em ambientes atuais (e.g. estuários de rios com intensa sedimentação, águas turvas, ambientes de transição). Aparentemente, as formas viventes apresentam relações ambientais similares ao contexto pré-histórico dos Sclerorhynchidae no estado do Maranhão.

\section{AGRADECIMENTOS}

Este trabalho foi possível graças ao apoio do Centro de Pesquisa em História Natural e Arqueologia do Maranhão (CPHNAMA), Laboratório de Entomologia e Vetores, (LEV), Laboratório de Estudos sobre as abelhas, (LEA) e Universidade Federal do Maranhão, (UFMA), pela disponibilização dos laboratórios e equipamentos utilizados para análise dos exemplares. Suporte financeiro: Coordenação de Aperfeiçoamento de Pessoal de Nível Superior (CAPES), Fundação de Amparo à Pesquisa e ao Desenvolvimento Científico e Tecnológico do Maranhão (FAPEMA).

\section{REFERÊNCIAS}

AMALFITANO, J.; GIUSBERTI, L.; DALLA VECCHIA; F.M. \& KRIWET, J. 2017. First skeletal remains of the giant sawfish Onchosaurus (Neoselachii, Sclerorhynchiformes) from the Upper Cretaceous of northeastern Italy. Cretac. Res, $69: 124-135$.

ARAMBOURG, C. 1940. Le groupe des Ganopristinés. B Soc Geol Fr, 10:127-147.

BRANCH, J.R. \& MOSLEY, J.L. 1997. The oldest sclerorhynchid sawfish (Rajiformes: Sclerorhynchidae) from the Lower Cretaceous of Texas. Tex. j. sci, 49:199-206.

CAPPETTA, H. 1974. Sclerorhynchidae nov fam, pristidae and pristiophoridae-example of parallelism among selacians. C R Hebd Seances Acad Sci, 278 : 225-228.

CAPPETTA, H. 1980. Les sélaciens du Crétacé supérieur du Liban. I: Requins. Palaeontogr Abt A, $68,69-148$.

CAPPETTA, H. 1987. Chondrichthyes II: mesozoic and cenozoic elasmobranchii. In: Cappetta, $\mathrm{H}$. Handbook of Paleontology, G. Fischer Verlag, 146157.

DUTRA, M.F.A \& MALABARBA, M.C.S.L. 2001.
Peixes do Albiano-Cenomaniano do Grupo Itapecuru no Estado do Maranhão, Brasil. In: Rossetti, D.F; Góes, A. M. \& Truckenbrodt, W. (eds.) O Cretáceo na Bacia de São Luís-Grajaú, 191-208.

FEITOSA, L.M.; LESSA, R.P.T.; MARTINS, A.P.B.; BARBIERI, R. \& NUNES, J.L.S. 2019. Daggernose Shark: An Elusive Species from Northern South America. Fisheries, 44: 144-147.

FEITOSA, L.M.; MARTINS, A.P.B. \& NUNES, J. L. S. 2017. Sawfish (Pristidae) records along the Eastern Amazon coast. Endanger. Species Res, 34:229-234.

FEITOSA, L.M.; MARTINS, A.P.B. \& Nunes, J.L.S. 2016. New record of Carcharhinus leucas (Valenciennes, 1839) in an equatorial river system. Mar. Biodivers. Rec, 9: 1-4.

FONTES, N. A.; MORAES, H. M. S. \& COSTA, S. A F. 2012. Ocorrência de Ischyrhiza (Batoide: Sclerorhynchidae) para o grupo Itapecuru, Cretáceo (Albiano) do estado do Maranhão, Brasil. Brazilian Geographical Journal: geosciences and humanities research medium, v. 3 , n. 1 .

GOMES, U.L; SILVA, R.F.L. \& SILVA, H.M.A. 2012. Elasmobranchii paleozoicos e mesozoicos na relação Brasil-África. In: Gallo, V.; Silva, H.M.A.; Brito, P.M. \& Figueiredo, F.J. (eds.) Paleontologia de vertebrados: relações entre América do Sul e África, Interciência, 21-61.

HAMMER, Ø., HARPER, D.A.T., RYAN, P.D. 2001. PAST: Paleontological statistics software package for education and data analysis. Palaeontologia Electronica 4(1): 9pp.

KEYES, I.W. 1977. Records of the northern hemisphere Cretaceous sawfish genus Onchopristis (Order Batoidea) from New Zealand. New Zeal J Geol Geop, 20:263-272.

KIRKLAND, J.I. \& MARTÍNEZ, M.C. 2002. Schizorhiza: a unique sawfish paradigm from the Difunta Group, Coahuila, Mexico. Rev Mex Cienc Geol, 19:16-24.

KLINGENBERG, C. P. 2016. Size, shape, and form: concepts of allometry in geometric morphometrics. Development Genes and Evolution, 226(3), 113-137. doi:10.1007/s00427-016-0539-2

KLEIN V.C. \& FERREIRA C.S. 1979. Paleontologia e Estratigrafia de uma fácies estuarina da Formação Itapecuru, Estado do Maranhão. An. Acad. Bras. Ciênc, 51: 523-533.

KRIWET, J. \& KUSSIUS, K. 2001. Paleobiology and paleobiogeography of sclerorhynchid sawfishes 
(Chondrichthyes Batomorphii). Rev Esp Paleontol, 35-46.

KRIWET, J. 2004. The systematic position of the Cretaceous sclerorhynchid sawfishes (Elasmobranchii, Pristiorajea. Mesozoic fishes, 3:57-73.

MCNULTY JR., C.L. \& SLAUGHTER, B. 1962. A new sawfish from the Woodbine Formation (Cretaceous) of Texas. Copeia, 4: 775-777.

MEDEIROS, M.A.; LINDOSO, R.M.; MENDES; I.D. \& CARVALHO; I.S. 2014. The Cretaceous (Cenomanian) continental record of the Laje do Coringa flagstone (Alcântara Formation), northeastern south America. J S AM Earth Sci, 53: 50-58.

PEDRÃO, E.; ARAI, M.; CARVALHO, IS. \& SANTOS M.H.B. 1993. Palinomorfos da Formação Itapecuru e análise palinológica de uma amostra de superfície da Ponta do Farol, São Luís-MA. CENPES/PETROBRAS, Relatório Técnico: 10.

PEREIRA A.A. \& MEDEIROS M.A. 2008. A new Sclerorhynchiform (Elasmobranchii) from the middle Cretaceous of Brazil. Rev Bras Paleontolog, 11: 207-212.

ROSSETTI, D.F. \& TRUCKENBRODT, W. 1997. Revisão estratigráfica para os depósitos do AlbianoTerciário Inferior (?) na Bacia de São Luís (MA), Bol. Mus. Para. Emilio Goeldi, Série Ciências da Terra, 9:29-4.

SCHAEFFER, B. 1963. Cretaceous fishes from Bolivia, with comments on pristid evolution. Am. Mus. Novit, 1-20.

SLAUGHTER, B.H \& SPRINGER, S. 1968. Replacement of rostral teeth in sawfishes and sawsharks. Copeia, 499-506.

SMITH, M.M.; RILEY,A.; FRASER, G.J.; UNDERWOOD, C.; WELTEN, M.; KRIWET,
J.; PFAFF, C. \& JOHANSON, Z. 2015. Early development of rostrum saw-teeth in a fossil ray tests classical theories of the evolution of vertebrate dentitions. Proc. Royal Soc. Lond B: Biological Sciences: 282.

STERNES, P.C.\& SHIMADA, K. 2018. Paleobiology of the Late Cretaceous sclerorhynchid sawfish, Ischyrhiza mira (Elasmobranchii: Rajiformes), from North America based on new anatomical data. Hist Biol, 1-18.

UNDERWOOD, C. J. 2006. Diversification of the Neoselachii (Chondrichthyes) during the Jurassic and Cretaceous. Paleobiology, 32: 215-235.

VICALVI M.A. \& CARVALHO I.S. 2002. Carófitas cretáceas da Bacia do Parnaíba (Formação Itapecuru), Estado do Maranhão, Brasil. In: $6^{\circ}$ Simpósio Sobre o Cretáceo no Brasil e $2^{\circ}$ Simpósio Sobre el Cretácico de América del Sur. 83-88.

WELTEN, M.; SMITH, M.M. \& UNDERWOOD, Z.J. 2015. Evolutionary origins and development of saw-teeth on the sawfish and sawshark rostrum (Elasmobranchii; Chondrichthyes). Royal Soc. Open Sci, 2:150-189.

WOSNICK, N.; PALMEIRA, A.R.O. \& NUNES, JORGE L.S. 2019. E-letter - Pinocch ioland: the role of the Brazilian Amazonian coast in elasmobranch conservation. Science (New York, N.Y.: Online), 334: 1-1.

WUERINGER, B. E.; PEVERELL, S. C.; SEYMOUR, J.; SQUIRE, L.; KAJIURA, S. M. \& COLLIN, S. P. 2011. Sensory systems in sawfishes 1. the ampullae of Lorenzini. Brain, behavior and evolution, 78:139-149.

WUERINGER, B. E.; SQUIRE; L. \& COLLIN, S. P. 2009. The biology of extinct and extant sawfish (Batoidea: Sclerorhynchidae and Pristidae). Rev Fish Biol Fisher, 19:445-464. 